\title{
educação

\section{Estilos intelectuais, estratégias de aprendizagem e adaptação acadêmica no ensino superior brasileiro}

\author{
Katya Luciane de Oliveira ${ }^{\mathrm{I}}$ \\ Universidade Estadual de Londrina, Brasil \\ Amanda Lays Monteiro Inácio \\ Universidade São Francisco, Brasil \\ Aline Oliveira Gomes da Silva ${ }^{\mathrm{III}}$, Maria Luzia \\ Silva Marianoiv \& Sandra Aparecida Pires \\ FRANCOV \\ Universidade São Francisco, Brasil
}

Objetivou-se no presente estudo investigar como se apresentam os estilos intelectuais, as estratégias de aprendizagem e a adaptação acadêmica no ensino superior, sendo buscadas possíveis correlações e relações de dependência entre essas variáveis. Participaram 396 estudantes de cursos de graduação de três estados do Brasil $(M=22,8$ anos, $D P$ $=4,9)$, de ambos os sexos, que responderam ao Inventário de Estilos Intelectuais/Pensamento - Revisado II (TSI-R2), à Escala de Estratégias de Aprendizagem (EEA-U) e ao Questionário de Adaptação Acadêmica do ensino Superior (QAES). Os resultados indicaram a prevalência do estilo hierárquico, das estratégias de autorregulação social, e ainda que os estudantes se encontram mais bem adaptados ao planejamento de carreira. Houve correlação positiva e significativa entre o estilo hierárquico e a adaptação ao planejamento de carreira, sendo esta de magnitude média, e entre esse mesmo estilo e as estratégias de autorregulação cognitivas e metacognitivas, de magnitude pequena. Foram encontradas relações de dependência entre o estilo hierárquico e a adaptação ao planejamento de carreira, e entre o estilo externo e a adaptação social. As implicações psicoeducacionais foram discutidas no sentido de conjecturar explicações mais profundas para as dificuldades dos estudantes do ensino superior.

Palavras-chave: Estilos de aprendizagem; Estratégias de aprendizagem; Adaptação acadêmica; Ensino superior

\section{N T R O D U Ç Ã O}

Tanto os estilos intelectuais quanto as estratégias de aprendizagem têm-se destacado em pesquisas como variáveis substanciais presentes no processo de aprendizagem e, consequentemente, na adaptação acadêmica dos estudantes (Purdie \& Hattie, 1996; Zhang, 2015; Zhang \& Sternberg, 2005; Zimmerman \& Martinez-Pons, 1986). Com base nisso, o presente estudo aborda os estilos intelectuais, as estratégias de aprendizagem e a adaptação acadêmica no ensino superior, buscando possíveis correlações entre as variáveis. 
Apesar da incipiência na literatura científica, as relações já encontradas evidenciam importantes contribuições, como a criação de medidas preventivas e remediativas por parte das instituições de ensino, para o desenvolvimento integral dos estudantes e o controle dos índices de evasão nesta etapa acadêmica (Oliveira, Santos, \& Inácio, 2018a).

$\mathrm{Na}$ perspectiva da Teoria do Autogoverno Mental proposta por Sternberg (1988) os estilos intelectuais apresentam-se como a expressão mais indicada no que concerne a questões atinentes a particularidades cognitivas e habituais, mediante as quais os estudantes processam as informações no decorrer da aprendizagem, considerados como diferentes padrões de processamento preferidos pelos estudantes no emprego do processamento da informação em meio à aprendizagem. Diante disso, cada estudante prefere um modo específico de organização cognitiva para receber e processar as informações novas e, assim, construir seu conhecimento. Ressalta-se que essa teoria embasa as concepções descritas anos mais tarde por Zhang e Sternberg (2005), que auxiliaram no aprimoramento da escala de estilos intelectuais utilizada no presente estudo, a saber, TSI-R2 (2007), advinda posteriormente às escalas TSI (1992) e TSI-R (2003) cronologicamente.

Segundo a Teoria do Autogoverno Mental, não existem estilos bons ou ruins; porém, alguns deles tornam-se mais eficazes, quando comparados a outros em determinados contextos. Assim, o mapeamento dos estilos intelectuais, frequentemente utilizados pelos estudantes, afigura-se como relevante à medida que compreendemos que esses estilos são modificáveis e aprendidos conforme a necessidade, podendo ser ainda utilizados, ou não, dependendo do contexto em que se apresentam (Fan \& Zhang, 2014; Oliveira, Trassi, \& Santos, 2017; Zhang, 2015).
Para identificar que estilos intelectuais são mais ou menos efetivos em determinados contextos, necessário se impõe compreender como tais estilos são classificados, quais suas características e em que momento este ou aquele estilo deve ser adotado no processamento da informação e/ou na execução de tarefas como melhor escolha. Assim, com base na teoria supracitada, são elencados treze estilos, a saber: legislativo, executivo, judiciário, global, local, liberal, conservador, hierárquico, monárquico, oligárquico, anárquico, interno e externo (Zhang, 2015; Zhang \& Sternberg, 2005).

O estilo legislativo se refere a preferência pelas próprias atividades de modo criativo, enquanto o executivo é comum aos que necessitam de regras claras e específicas. O estilo judicial diz respeito à primazia do sujeito por avaliar os demais, enquanto o hierárquico é característico daqueles que dão atenção a várias tarefas priorizando-as segundo sua importância. O estilo monárquico engloba aqueles que elegem uma tarefa por vez; já o oligárquico, é mais comum em indivíduos que gostam de realizar diversas atividades ao mesmo tempo sem o estabelecimento de prioridades. O estilo anárquico se refere à preferência por tarefas flexíveis. O global é comum aos que elegem tarefas abstratas e sem detalhes; enquanto isso, o estilo local tem como característica o uso de ideias concretas detalhadas. O estilo interno é característico daqueles que preferem trabalhar sozinhos, e o externo daqueles com preferência por trabalhar em grupo. Por fim, o estilo liberal diz respeito à prioridade por atividades que envolvam coisas novas e o estilo conservador pelo estabelecimento de regras e procedimentos fixos (Fan, 2016; Zhang \& Sternberg, 2005).

Esses estilos podem ser classificados em três tipos, denominados I, II e III. O Tipo I, ou cognitivo, é caracterizado por agregar 
os estilos que envolvem criatividade $\mathrm{e}$ complexidade cognitiva na preferência do estudante e caracteriza-se por seu elevado caráter adaptativo. Nele estão agrupados os estilos legislativo, judicial, liberal, global e hierárquico. O Tipo II, ou desempenho típico, refere-se à preferência pelo cumprimento de regras e procedimentos fixos, caracterizado também por menor complexidade cognitiva. Esse tipo abarca os estilos executivo, conservador, local e monárquico. O Tipo III, ou focado na atividade, reúne elementos de ambos os tipos anteriormente mencionados, dependendo das escolhas do estudante em cada contexto de estudo. Os estilos que fazem parte desse tipo são: oligárquico, anárquico, interno e externo (Zhang, 2015).

As pesquisas realizadas concentramse, sobretudo, no ensino superior (Cheng, Zhang, \& Hu, 2016; Fan, 2016; Fan \& Zhang, 2014; Fan, Zhang, \& Chen, 2018; Zhang, 2015), sendo em menor escala encontradas em relação ao ensino fundamental (Oliveira, Inácio, \& Buriolla, 2016; Trassi, 2016; Wang \& Tsent, 2014) e médio (Oliveira, Inácio, Santos, Mariano, \& Franco, 2017; Yuan, Zhang, \& Fu, 2017). Esses estudos são mais frequentemente encontrados na literatura internacional, embora existam precursores no contexto brasileiro, que contribuem substancialmente para o mapeamento e para a compreensão dos estilos utilizados por estudantes em diferentes etapas de ensino, a fim de aprimorar o desenvolvimento das habilidades e o desempenho dos discentes.

Aliados às contribuições elucidadas, o desenvolvimento da autonomia e a melhoria do desempenho acadêmico na identificação e uso das estratégias de aprendizagem também podem contribuir para que esse estudante aprenda a posicionar-se de forma ativa $e$ consciente diante dos processos envolvidos na aquisição de novos conhecimentos
(Boruchovitch, 1999). As estratégias de aprendizagem são concebidas como métodos e procedimentos a que os estudantes recorrem para adquirir informação e potencializar seus processos de aprendizagem. Para desenvolver os estudos sobre as estratégias, pesquisas realizadas (Boruchovitch, 1999; Purdie \& Hattie, 1996; Zimmerman \& Martinez-Pons, 1986) objetivam a compreensão de como os estudantes escolhem, obtêm e retêm as novas informações.

Dentre as diversas categorizações para compreensão das estratégias de aprendizagem, o presente estudo norteia-se pela concepção de que tais estratégias posicionam-se em dois grandes grupos: o de estratégias cognitivas e o de estratégias metacognitivas (Dembo, 1994; Weinstein, Acee, \& Jung, 2011). O primeiro consiste no auxílio direto que as informações oferecem. Trata-se de atividades mais específicas que podem ser subdivididas em estratégias de ensaio (repetir, copiar, sublinhar), estratégias de elaboração (resumir, criar analogias, responder a perguntas) e estratégias de organização (selecionar ideias, fazer esquemas e mapas). As estratégias metacognitivas apresentam maior grau de complexidade e concentram-se no controle e no planejamento dos processos cognitivos, tais como: estabelecimento de metas, organização do ambiente, escolha e testagem de estratégias para cada contexto, entre outras.

Pesquisas no Brasil (Alcará \& Santos, 2015; Boruchovitch \& Santos, 2015; Cunha \& Boruchovitch, 2016; Ganda \& Boruchovitch, 2015) evidenciam que as estratégias de aprendizagem impactam de forma considerável o processo de aprendizagem e, consequentemente, o rendimento dos estudantes. Isso porque, quando conhecem e entendem seus processos cognitivos, esses alunos conseguem raciocinar sobre seu próprio pensamento, selecionar estratégias 
mais eficazes ao seu contexto e aprimorar sua performance e rendimento, a fim de construir o conhecimento com maior facilidade.

No âmbito do ensino superior, para além do mapeamento dos estilos intelectuais e do uso de estratégias de aprendizagem adequadas, faz-se necessário analisar construtos como a adaptação acadêmica do estudante, posto que o ingresso nessa etapa de ensino corresponde a um período de grandes expectativas e sentimentos positivos pela realização de uma importante conquista, mas que traz em sua esteira inúmeras exigências para o êxito nesse novo contexto. A universidade é um campo fértil para a aprendizagem de novas habilidades que extrapolam os conhecimentos acadêmicos e demandam do aluno certo ajustamento a essa nova realidade (Igue, Bariani, \& Milanesi, 2008; Soares \& Del Prette, 2015; Tomás, Ferreira, Araújo, \& Almeida, 2014). A complexidade que envolve essa etapa pode ser compreendida sob diferentes perspectivas teóricas, dentre as quais se elege aquelas que valorizam, de modo substancial, a contribuição do ambiente institucional e social, com os quais o estudante vai interagir durante seu processo de adaptação, o que determinará, por sua vez, a permanência ou o abandono do curso (Astin, 1993; Tinto, 1993). A adaptação acadêmica refere-se à capacidade de o aluno integrar-se no ensino superior, fazendo uso de seus processos cognitivos, sociais e afetivos, que, juntos, possibilitam ganhos para a aprendizagem e, sobretudo, para a formação profissional (Oliveira et al., 2018a; Santos, Oliveira, \& Dias, 2015). Ao se considerar que essa etapa corresponde ao processo de transição entre a adolescência e a fase adulta, a adaptação deve ser avaliada em perspectiva desenvolvimentista. Nesse contexto, o suporte social recebido, que corresponde ao apoio de familiares, amigos antigos e novos colegas do meio acadêmico, insere-se como um aspecto inerente à adaptação acadêmica e que se reveste de fundamental importância; isso porque os relacionamentos interpessoais, como um todo, contribuem não apenas para a adaptação acadêmica do estudante, mas, sobretudo, para adiar ou confirmar a decisão de esse estudante permanecer ou abandonar o curso (Araújo et al., 2016; Santos et al., 2015, Tomás et al., 2014).

Soares e Del Prette (2015) apontam as habilidades e as competências sociais como variáveis correlacionadas com o ajustamento ao ensino superior, uma vez que atuam como facilitadoras para a vivência das diversas experiências, assim como contribuem para uma melhor satisfação pessoal e um maior índice de comprometimento com o aprendizado. Uma adaptação bem-sucedida implica ainda o envolvimento do estudante com atividades curriculares e extracurriculares e com a persistência assumida nos momentos de dificuldade. Contudo, tais fatores podem ser influenciados pela qualidade das redes sociais formadas, que contribuem para o amparo e compartilhamento das situações vivenciadas, para a satisfação pela escolha profissional e para os índices de evasão acadêmica (Ambiel \& Barros, 2018; Tomás et al., 2014).

Diante do exposto, Igue et al. (2008) sugerem que a criação de programas de integração contribui para que os estudantes de graduação recém-chegados percebam os diversos serviços oferecidos pela instituição e ainda enseja a socialização entre ingressantes e veteranos, contribuindo igualmente para a construção de uma visão mais comprometida com a realidade. Desse modo, as instituições podem fazer uso do mapeamento dos estilos intelectuais e promoção das estratégias de aprendizagem como facilitadores da aquisição do conhecimento. Por consequência, o ensino superior deve ocupar-se, para além dos conhecimentos científicos adquiridos ao longo 
do curso, com a formação integral do estudante que irá atuar ativamente na sociedade por meio da profissão escolhida (Santos, Polydoro, Scortegagna, \& Linden, 2013).

Ademais, será importante considerar os desafios relacionados com as diferentes dimensões do ensino superior, ou seja, adaptação ao estudo, adaptação institucional, social, pessoal-emocional, e o planejamento de carreira. As mudanças no que concerne ao estudo e ao meio institucional relacionam-se com uma maior autonomia e responsabilidade pelas atividades acadêmicas, que se tornam mais complexas no ensino superior. As transformações sociais envolvem novas formas de relacionamento com a família, sobretudo para aqueles que ingressam em instituições em outra cidade, com novos colegas, professores e outras figuras de autoridade. Por sua vez, as mudanças pessoais-emocionais e o planejamento de carreira dizem respeito ao desenvolvimento da identidade profissional do estudante, seu comprometimento com o curso e gestão de recursos financeiros (Carlotto, Teixeira, \& Dias, 2015; Soares, Pinheiro, \& Canavarro, 2015).

O estudo de Oliveira et al. (2018a), a respeito da adaptação acadêmica e dos estilos intelectuais, buscou averiguar possíveis diferenças quanto ao curso escolhido e relações entre as variáveis. Assim, os resultados evidenciaram a existência de diferenças significativas nas dimensões: planejamento de carreira, adaptação pessoal-emocional, adaptação institucional e total, e nos cursos de graduação, sendo que o curso de Psicologia obteve pontuações mais elevadas em todas as dimensões, indicando que esses estudantes demonstram mais autoconhecimento acerca de suas escolhas e motivações. Ademais, as correlações encontradas entre a adaptação e os estilos foram em grande parte significativas, indicando consistência teórica na discriminação dessas variáveis e no modo como se relacionam.

A pesquisa de Oliveira et al. (2018a), ora citada, evidencia os primeiros esforços nacionais de associação entre a adaptação acadêmica e os estilos intelectuais. Contudo, destacam-se outros estudos presentes na literatura mais recente, que buscam associar a adaptação acadêmica a variáveis diversas, tais como: autoconsciência da aparência (Mendes et al., 2018), evasão e satisfação com a escolha profisisonal (Ambiel \& Barros, 2018), adaptação acadêmica e coping (Carlotto et al., 2015), sendo os aspectos relacionados à aprendizagem em si incipientemente abordados. No intuito de contribuir para a área psicoeducacional, sobretudo no que tange ao ensino superior, a presente pesquisa, quantitativa, de análise descritiva e correlacional, objetivou investigar como se apresentam os construtos estilos intelectuais, estratégias de aprendizagem e adaptação acadêmica no ensino superior, buscando possíveis correlações e relações de dependência entre essas variáveis.

\section{MÉ T O D O \\ 1. 1 PARTICIPANTES}

Participaram 396 estudantes de graduação escolhidos por amostra não probabilística por conveniência. Destes, 44,7\% $(n=177)$ eram provenientes do ensino particular e 55,3\% $(n=219)$ do ensino público de três estados brasileiros, a saber: Paraná $(24,7 \%, n=98)$, São Paulo $(29 \%, n=115)$ e Rio Grande do Sul $(46,2 \%, n=183)$. A média de idade foi de 22 anos e 8 meses $(D P=4,9)$, sendo a idade mínima de 17 anos e a máxima 49 anos. O sexo masculino representou 44,2\% $(n=175)$ e o feminino $54,8 \%(n=217)$, sendo que $1,0 \%$ não foi informado $(n=4)$. Quanto ao curso dos 
estudantes, 13,6\% ( $n=54)$ eram de Psicologia, 11,9\% ( $n=47)$ de Ciências Contábeis, 20,7\% $(n=82)$ de Letras, 23\% ( $n=91)$ de História, $11,4 \%(n=45)$ de Agronomia, 12,6\% ( $n=50)$ de Odontologia, e 6,8\% $(n=27)$ do curso de Engenharia. Os participantes pertenciam a semestres distintos da graduação. No entanto, este dado não foi levantado por não fazer parte dos objetivos do estudo.

\subsection{INSTRUMENTOS}

Inventário de Estilos Intelectuais/Pensamento - Revisado II (TSI-R2) - (Sternberg, Wagner, \& Zhang, 2007)

De origem norte-americana, o inventário foi proposto inicialmente por Sternberg e Wagner (1992), contendo 104 itens organizados nos 13 estilos intelectuais. A primeira revisão foi realizada por Sternberg, Wagner, e Zhang (2003) (TSI-R), em que alguns itens foram suprimidos, tendo essa nova organização 65 itens. A versão mais recente, elaborada por Sternberg et al. (2007), e denominada (TSI-R2), mantém a organização proposta anteriormente, com 13 estilos, sendo a mais frequente encontrada na literatura e utilizada no presente estudo. O TSI-R2 é composto por 65 itens, dispostos em escala do tipo Likert de sete pontos, quais sejam: "De jeito nenhum" (1 ponto), "Não muito bem" ( 2 pontos), "Um pouco" (3 pontos), "Bem de alguma forma" (4 pontos), "Bem" (5 pontos), "Muito bem" (6 pontos) e "Extremamente bem" (7 pontos) (Zhang, 2011). O instrumento não apresenta um escore total, pois cada estilo intelectual deve ser considerado como independente. De aplicação individual ou coletiva, o inventário possui estudos de validade para a realidade norte-americana, com coeficientes de alfa de Cronbach que variam de 0,70 a 0,80 (Zhang, 2011) e evidências de validade de conteúdo para o Brasil (Oliveira, Santos, \& Inácio, 2018b).

Escala de Estratégias de Aprendizagem para Universitários - EEA-U - (Boruchovitch \& Santos, 2015)

A escala é composta por 35 itens, organizados em uma escala do tipo Likert, que corresponde à maneira como os estudantes costumam estudar ou se preparar para uma avaliação acadêmica. As questões apresentam quatro alternativas de respostas que variam entre "sempre" ( 3 pontos), "às vezes" ( 2 pontos), "raramente" (1 ponto) e "nunca" (0 pontos), perfazendo um total de 105 pontos. Esta escala apresenta uma consistência interna considerada boa, com um alfa de Cronbach de 0,87 para a sua totalidade. Identificou-se também a existência de três fatores, quais sejam: autorregulação cognitiva e metacognitiva, com 23 itens e $\alpha=0,86$; autorregulação dos recursos internos e contextuais, com 8 itens e $\alpha=0,71$; e autorregulação social, com 4 itens e $\alpha=0,65$.

Questionário de Adaptação ao Ensino Superior (QAES) - (Araújo et al., 2014)

$\mathrm{O}$ instrumento averigua a adaptação de estudantes ao ensino superior. Proposto inicialmente com 68 itens, os estudos de validade realizados com graduandos portugueses apontaram a existência de seis dimensões com indicadores psicométricos adequados. No entanto, investigações posteriores com estudantes de outros países sugeriram a necessidade de uma nova organização. Dessa feita, o instrumento em questão adaptado à realidade brasileira por Araújo et al. (2014) possui 40 itens dispostos em cinco dimensões (oito itens por dimensão) teoricamente consistentes, com índices de consistência interna considerados adequados, acima de 0,70 . Os itens são apresentados em 
escala Likert que varia entre 1 (Discordo totalmente) e 5 (Concordo totalmente).

\subsection{PROCEDIMENTOS}

Diante das autorizações das instituições envolvidas e a aprovação do Comitê de Ética em Pesquisa com Seres Humanos - CEP da Universidade Estadual de Londrina, a coleta de dados foi realizada. Todos os procedimentos éticos foram seguidos e estão de acordo com a Resolução 510/2016 do Conselho Nacional de Saúde/Brasil e seus complementares, sendo o critério para inclusão dos participantes a assinatura do Termo de Consentimento Livre e Esclarecido - TCLE por aqueles maiores de 18 anos e, além disso, a assinatura dos responsáveis pelos estudantes menores de idade. Foram agendados dia e horário para cada uma das universidades participantes, sendo os instrumentos aplicados coletivamente, em sala de aula, na mesma ordem, a saber: Inventário de Estilos Intelectuais/Pensamento - Revisado II (TSI-R2), Escala de Estratégias de Aprendizagem para Universitários - EEA-U e Questionário de Adaptação ao Ensino Superior (QAES), com duração aproximada de 50 minutos por turma.

\subsection{CONSIDERAÇÕ ES ÉTICAS}

As respostas dos discentes foram organizadas em planilha eletrônica e recorreu-se ao método de análise das estatísticas descritiva einferencial por meio do Programa IBM-Statistical Package for the Social Sciences for Windows $($ versão 22.0 - SPSS. Inicialmente, realizou-se uma análise descritiva para verificar as médias, desvios padrão e pontuações mínima e máxima obtidas. Na sequência, realizou-se a análise de correlação de Pearson entre os escores dos instrumentos para buscar possíveis relações. Por fim, utilizou-se a análise de regressão linear simples pelo método enter, a fim de identificar possíveis relações de dependência entre os estilos intelectuais, as estratégias de aprendizagem e a adaptação acadêmica dos estudantes.

\section{RESULTADOS}

Os resultados serão apresentados na sequência, conforme descrição dos objetivos propostos. No que se refere à averiguação dos estilos intelectuais, das estratégias de aprendizagem e da adaptação acadêmica, as Tabelas 1, 2 e 3 apresentam os resultados descritivos das médias, desvios padrão, pontuações mínimas e máximas obtidas. A Tabela 3 indica ainda a média ponderada de pontos, em razão das subescalas possuírem quantidades distintas de itens. Assim, a média de cada subescala foi dividida pelo número de itens, fornecendo uma comparação adequada da pontuação obtida pela amostra.

Mediante os resultados apresentados na Tabela 1, foi possível observar que o estilo com a média mais elevada foi o hierárquico, seguido pelos estilos externo, legislativo e judicial. Em contrapartida, os estilos que apresentaram médias mais baixas foram, respectivamente, o oligárquico, o local e o global.

Conforme os resultados apresentados na Tabela 2, observou-se que a subescala de Autorregulação Social obteve a média mais elevada. Destacase que nessa tabela os resultados devem ser examinados de acordo com a média ponderada, tendo em consideração que a quantidade de itens é diferente entre as subescalas.

De acordo com os resultados da Tabela 3, observou-se que a subescala de adaptação ao Planejamento de Carreira foi a mais pontuada pelos estudantes da amostra. Por outro lado, a menos pontuada foi a subescala de Adaptação Pessoal-Emocional.

Com a finalidade de analisar as correlações entre os construtos avaliados, a Tabela 4 
TA B EL A 1

Estatística Descritiva dos Estilos Intelectuais

\begin{tabular}{lcccc}
\hline \multicolumn{1}{c}{ Estilos } & Médias & Desvio padrão & $\begin{array}{c}\text { Pontuações } \\
\text { Mínima e } \\
\text { Máxima }\end{array}$ & $\begin{array}{c}\text { Variação da } \\
\text { Pontuação }\end{array}$ \\
\hline Legislativo & 24,65 & 4,86 & $11-35$ & $5-35$ \\
Executivo & 22,71 & 3,72 & $11-32$ & $5-35$ \\
Judicial & 24,58 & 3,79 & $14-34$ & $5-35$ \\
Global & 21,84 & 3,69 & $9-33$ & $5-35$ \\
Local & 21,61 & 4,67 & $7-33$ & $5-35$ \\
Liberal & 23,86 & 5,47 & $10-35$ & $5-35$ \\
Conservador & 23,28 & 4,59 & $9-35$ & $5-35$ \\
Hierárquico & 25,95 & 3,92 & $16-35$ & $5-35$ \\
Monárquico & 22,20 & 4,20 & $10-34$ & $5-35$ \\
Oligárquico & 21,38 & 4,88 & $7-33$ & $5-35$ \\
Anárquico & 22,86 & 4,65 & $12-35$ & $5-35$ \\
Interno & 23,53 & 4,48 & $9-34$ & $5-35$ \\
Externo & 24,81 & 4,07 & $14-35$ & $5-35$ \\
\hline
\end{tabular}

TA B E L A 2

Estatística Descritiva das Estratégias de Aprendizagem

\begin{tabular}{lccccc}
\hline \multicolumn{1}{c}{ Estratégias } & Média & $\begin{array}{c}\text { Desvio } \\
\text { padrão }\end{array}$ & $\begin{array}{c}\text { Média } \\
\text { ponderada }\end{array}$ & $\begin{array}{c}\text { Pontuações } \\
\text { Mínima e } \\
\text { Máxima }\end{array}$ & $\begin{array}{c}\text { Variação da } \\
\text { Pontuação }\end{array}$ \\
\hline Autorregulação 1 & 44,23 & 6,03 & 1,92 & $30-66$ & $3-69$ \\
Autorregulação 2 & 14,36 & 4,87 & 1,79 & $4-41$ & $3-24$ \\
Autorregulação 3 & 8,86 & 1,72 & 2,21 & $4-12$ & $3-12$ \\
EA Total 4 & 71,66 & 8,98 & 1,79 & $49-96$ & $3-105$ \\
\hline Nota. 1 = Autorregulação Cognitiva e Metacognitiva; 2 = Autorregulação dos Recursos \\
Internos e Contextuais; 3 = Autorregulação Social; 4 = Estratégias de Aprendizagem Total
\end{tabular}

apresenta as correlações encontradas entre os estilos intelectuais e a adaptação ao ensino superior. Posteriormente, a Tabela 5 refere-se às demais correlações encontradas.

Os resultados da Tabela 4 indicam a existência de correlações de magnitude pequena e média (segundo a classificação de Cohen, 1988) entre os estilos intelectuais e duas das subescalas do questionário de adaptação ao ensino superior, a saber: adaptação ao Planejamento de Carreira e Adaptação Social. A correlação mais elevada encontra-se entre o estilo hierárquico e a subescala de adaptação ao Planejamento de Carreira.

De acordo com os dados apresentados na Tabela 5 , houve correlações positivas e negativas entre os estilos intelectuais e a adaptação ao ensino superior com as estratégias de aprendizagem. 
TAB ELA 3

Estatística Descritiva da Adaptação ao Ensino Superior

\begin{tabular}{lcccc}
\hline \multicolumn{1}{c}{ Subescalas Adaptação } & Média & $\begin{array}{c}\text { Desvio } \\
\text { padrão }\end{array}$ & $\begin{array}{c}\text { Pontuações } \\
\text { Mínima e } \\
\text { Máxima }\end{array}$ & $\begin{array}{c}\text { Variação de } \\
\text { Pontuação }\end{array}$ \\
\hline Planejamento de Carreira & 32,10 & 7,73 & $8-40$ & $5-40$ \\
Social & 31,48 & 9,32 & $11-40$ & $5-40$ \\
Pessoal-Emocional & 26,18 & 4,90 & $12-40$ & $5-40$ \\
Estudo & 27,39 & 4,36 & $12-40$ & $5-40$ \\
Institucional & 27,29 & 4,90 & $11-40$ & $5-40$ \\
Adaptação Total & 155,02 & 16,91 & $61-198$ & $1-200$ \\
\hline
\end{tabular}

TAB ELA 4

Estatística Descritiva da Adaptação ao Ensino Superior

\begin{tabular}{lcc}
\hline \multicolumn{1}{c}{ Estilos } & $\begin{array}{c}\text { Adaptação ao } \\
\text { Planejamento de Carreira }\end{array}$ & Adaptação Social \\
\hline Legislativo & $0,274^{* *}$ & $0,157^{*}$ \\
Judicial & $0,221^{*}$ & $0,225^{*}$ \\
Global & $0,121^{*}$ & $0,223^{*}$ \\
Local & $0,397^{* *}$ & $0,180^{*}$ \\
Hierárquico & $0,264^{* *}$ & $0,281^{* *}$ \\
Anárquico & $0,182^{* *}$ & $0,181^{* *}$ \\
Externo & $0,389^{* *}$ \\
\hline Nota. Nível de significância ${ }^{*}=0,050 ; * *=0,01 ; * * *=0,001$
\end{tabular}

TABELA 5

Correlações entre Estilos Intelectuais e Adaptação ao Ensino Superior com as Estratégias de Aprendizagem

\begin{tabular}{lccc}
\hline \multicolumn{1}{c}{ Estilos } & $\begin{array}{c}\text { Autorregulação } \\
\text { Social }\end{array}$ & $\begin{array}{c}\text { Autorregulação } \\
\text { Cognitiva e } \\
\text { Metacognitiva }\end{array}$ & $\begin{array}{c}\text { Autorregulação dos } \\
\text { Recursos Internos e } \\
\text { Contextuais }\end{array}$ \\
\hline Externo & $0,166^{*}$ & $0,230^{*}$ & \\
Hierárquico & $0,227^{*}$ & \\
Legislativo & $0,217^{*}$ & $0,148^{*}$ \\
Judicial & & \\
Adaptação Social & $-0,225^{*}$ & \\
\hline Nota . Nível de significância $*=0,050 ; * *=0,01 ; * * *=0,001$ &
\end{tabular}


As maiores correlações encontradas foram entre os estilos hierárquico, legislativo e judicial e a subescala de Autorregulação Cognitiva e Metacognitiva; no entanto, todas de magnitude pequena (segundo a classificação de Cohen, 1988).

Para verificar a relação de dependência entre os construtos estudados, recorreu-se à análise de regressão linear. Os dados indicaram que o estilo hierárquico demonstrou prever em aproximadamente $11 \%$ a adaptação ao Planejamento de Carreira, considerando $\mathrm{R}=0,397, \quad \mathrm{R}^{2}$ ajustado=0,111; $\mathrm{F}(1,362)=23,202 ;$ Beta $=0,397, t=12,80$ e $p=0,001$. Desse modo, infere-se que, até certo ponto, estudantes que preferem realizar suas atividades de forma mais hierárquica e ordenada tendem a compreender melhor suas intenções e percepções acerca do curso escolhido.

Ademais, o estilo externo pôde prever em aproximadamente $11 \%$ a Adaptação Social da amostra pesquisada, considerando $\mathrm{R}=0,389$, $\mathrm{R}^{2}$ ajustado $=0,113 ; \mathrm{F}(1,374)=23,202 ;$ Beta $=0,389$, $t=7,40$ e $p=0,001$. Assim, os estudantes do ensino superior com preferência por realizar tarefas em grupo tendem a estar mais bem adaptados socialmente, o que indica maior abertura no relacionamento estabelecido com os colegas.

\section{DIsCUSS Ão}

Considerando os objetivos do presente estudo, constatou-se que os estudantes do ensino superior da amostra pesquisada obtiveram melhores médias nos estilos: hierárquico, externo, e legislativo judicial. Três desses estilos concentram-se no Tipo I, que se refere a um processamento cognitivo mais complexo e criativo (Fan, 2016; Fan \& Zhang, 2014; Zhang, 2015). As características inerentes a esses estilos mais pontuados correspondem a um perfil de aluno que estabelece prioridades em seus estudos, gosta de trabalhar de modo coletivo, é criativo e avaliativo quanto ao seu próprio desempenho e dos demais. De modo geral, estas são características facilmente reconhecidas no ensino superior, em que principalmente a alta demanda de atividades exige do aluno uma maior complexidade cognitiva na execução das tarefas e, ainda, que os estudantes hierarquizem suas tarefas, realizando-as segundo o grau de importância. Além disso, o estabelecimento de graus de prioridade possibilita que o estudante se organize melhor e possa, consequentemente, atribuir a atenção devida a cada uma das atividades a serem executadas (Oliveira et al., 2018a; Santos et al., 2015; Soares \& Del Prette, 2015).

Ainda em relação aos estilos intelectuais, destaque-se que o estilo menos pontuado foi o oligárquico, que se refere à preferência por trabalhar em várias tarefas ao mesmo tempo, sem o estabelecimento de prioridades (Zhang \& Sternberg, 2005). Assim, há consistência teórica nos dados apresentados pela amostra, considerando-se que esta descrição de estilo se opõe ao resultado mais proeminente encontrado. Tendo em vista que os estudos acerca dos estilos intelectuais ainda se encontram em fase inicial em contexto brasileiro, tal resultado agrega embasamento ao evidenciar essa oposição.

No tocante às estratégias de aprendizagem, a média mais elevada foi em relação à Autorregulação Social, que se refere à socialização de ajuda aos colegas em caso de dúvidas e estudo em grupo, entre outros (Boruchovitch \& Santos, 2015). Esse dado mostra-se consoante com o anterior, no qual o estilo externo se mostrou como preponderante entre os estudantes da amostra. Assim, supõe-se que esses estudantes tenham preferência por atividades em grupo e se mostrem autorregulados socialmente. A segunda subescala mais pontuada foi a de Autorregulação Cognitiva e Metacognitiva, que se relaciona com a capacidade de o discente processar tanto informações mais gerais, quanto 
processar e compreender conteúdos que exijam automonitoramento e possível autorregulação. Tendo em vista que o uso dessas estratégias se mostra determinante para o desempenho acadêmico dos estudantes, atuando como facilitador para que a aprendizagem ocorra (Boruchovitch, 1999; Zimmerman \& MartinezPons, 1986), pode-se afirmar que os estudantes da amostra pesquisada encontram-se em nível do esperado (desejável) no que tange ao uso dessas estratégias, o que se mostra consonante com a literatura da área (Alcará \& Santos, 2015; Boruchovitch \& Santos, 2015).

A adaptação ao ensino superior evidenciou-se adequada, estando a média de acertos acima da metade dos pontos possíveis, aproximadamente $77,5 \%$. Sendo assim, os estudantes da amostra pesquisada mostram-se adaptados ao ensino superior de modo geral. Quanto às subescalas do questionário, a maior pontuação foi em relação ao Planejamento de Carreira, indicando a existência de satisfação com o curso e com a escolha profissional realizada. Os estudantes do ensino superior também se mostram adaptados no que tange à perspectiva social (Adaptação Social), que se refere ao relacionamento e envolvimento com os colegas do curso. De modo geral, esse resultado encontra-se de acordo com a literatura acerca do tema, na medida em que Oliveira et al. (2018a) também encontraram médias mais altas nessas subescalas em seu estudo, com 628 estudantes provenientes de quatro universidades de diferentes estados brasileiros.

No que concerne às correlações encontradas entre os estilos intelectuais e a subescala adaptação ao Planejamento de Carreira, correspondente ao Questionário de Adaptação ao ensino superior, as mais altas foram nos estilos hierárquico e judicial. Assim, tem-se que os estudantes mais bem adaptados ao curso escolhido tendem a fazer uso de estilos do Tipo I, que correspondem a um caráter adaptativo mais alto e certa complexidade cognitiva (Fan, 2016). Estudantes que preconizam estilos desse tipo tendem a agir mais livre e criativamente, conseguindo adaptarse com mais facilidade a novos contextos, o que explica a correlação encontrada com a Adaptação de Carreira, pois esses alunos tendem a estudar e a planejar seus ideais, mas lidam com mudanças de forma mais positiva quando comparado aos demais tipos. Tendo em vista essas características, e ainda diversos estudos da área, pode-se dizer que o uso de estilos do Tipo I contribui para uma aprendizagem mais efetiva (Fan, 2016; Fan \& Zhang, 2014; Zhang \& Sternberg, 2005).

Ainda no tocante às correlações, vale destacar a correlação negativa encontrada entre o estilo externo e a Adaptação Social dos estudantes da amostra pesquisada. Apesar de, até ao presente momento, não serem encontrados outros estudos com os quais tal dado pudesse ser comparado, pressupõe-se que uma Adaptação Social bem estabelecida poderia contribuir para que o aluno dependente dos colegas de turma pudesse sentirse menos autorregulado para aprender por conta própria; sugere-se, por esse motivo, a realização de outros estudos que possam averiguar com maior profundidade os resultados até ao momento encontrados.

Por fim, quanto às análises de regressão realizadas, constatou-se que o estilo hierárquico pode prever, em certa medida, o Planejamento de Carreira dos estudantes; por seu lado, o estilo externo previu, em parte, a Adaptação Social. Esses resultados evidenciam relações de dependência importantes, pois, à medida que o estudante consegue organizar suas atividades e priorizá-las segundo sua importância, tende também a planejar melhor seus objetivos e planos relacionados ao curso. Além disso, os discentes, que têm preferência por atividades coletivas e apreciam o contato com outras pessoas, mostram-se mais adaptados socialmente ao curso.

Faz-se necessário reafirmar a importância de que as universidades promovam apoio 
institucional e integração de seus alunos, fornecendo meios para que esses estudantes possam alinhar suas expectativas à realidade (Igue et al., 2008), quer organizando-se de maneira adequada para lidar com exigências mais complexas que fazem parte dessa nova etapa de ensino, quer identificando e fazendo uso de meios, como os estilos intelectuais e as estratégias de aprendizagem enquanto atributos facilitadores para a aquisição do conhecimento (Oliveira et al., 2018a; Santos et al., 2015).

A compreensão das diferenças no modo como cada aluno faz uso de suas habilidades no momento do estudo permite não apenas a criação de explicações mais profundas acerca da performance desse sujeito, mas também traz contribuições para sua adaptação ao ambiente de aprendizagem (Oliveira et al., 2016; Oliveira et al., 2017; Zhang, 2015). Também o conhecimento dessas variáveis possibilita a criação de medidas de prevenção e, até mesmo, remediação das dificuldades acadêmicas (Oliveira et al., 2018a).

Ao se considerar que os construtos estudados carecem de dados mais representativos para comparações entre as realidades contextuais aos quais se aplicam, aponta-se a necessidade de lembrar as limitações do presente estudo. A esse respeito, ressalte-se que os participantes eram provenientes de apenas três estados brasileiros, sendo necessária uma amostra mais abrangente, a fim de se alcançar um mapeamento balizado quanto aos estilos intelectuais, estratégias de aprendizagem e de adaptação acadêmica do estudante do ensino superior brasileiro em sentido mais amplo, buscando até mesmo diferenças que considerem gênero, modalidade de ensino particular e público, condições socioeconômicas e momento do curso.

Ressalta-se ainda que as relações de predição encontradas entre as variáveis do presente estudo são relativamente limitadas; no entanto, ao considerar a subjetividade presente na área das ciências humanas, identificam-se esses índices como importantes a serem apresentados. Posto isso, há desafios a serem vencidos para que o estudante do ensino superior possa dispor de uma formação que, de fato, o prepare, o torne capaz de enfrentar o futuro, e possa ingressar no mercado de trabalho marcando seu desempenho como competente contribuição na sociedade em que atuar.

\section{REFERENCIAS}

Alcará, A. R., \& Santos, A. A. A. (2015). Avaliação e desenvolvimento da compreensão de leitura em universitários. Estudos de Psicologia, 32(1), 63-73. DOI: 10.1590/0103-166X2015000100006

Ambiel, R. A. M., \& Barros, L. O. (2018). Relações entre evasão, satisfação com a escolha profissional, renda e adaptação de universitários. Psicologia: Teoria e Prática, 20(2), 254-267. DOI: 10.5935/1980-6906/ psicologia.v20n2p254-267

Araújo, A. M., Almeida, L. S., Ferreira, J. A., Santos, A. A., Noronha, A. P., \& Zanon, C. (2014). Questionário de Adaptação ao Ensino Superior (QAES): Construção e validação de um novo questionário. Psicologia, Educação e Cultura, 18(1), 131-145. Disponível em https://www. researchgate.net/publication/268148778 Questionario de Adaptacao ao Ensino Superior QAES Construcao e validacao de um novo questionario

Araújo, A. M., Santos, A. A., Noronha, A. P., Zanon, C., Ferreira, J. A., Casanova, J. R., \& Almeida, L. S. (2016). Dificuldades antecipadas de adaptação ao ensino superior: Um estudo com alunos do primeiro ano. Revista de Estudios e Investigación en Psicología y Educación, 3(2), 102-111. DOI: 10.17979/reipe.2016.3.2.1846

Astin, A. (1993). What matters in college? Four critical years revisited. San Francisco: Jossey Bass. 
Boruchovitch, E. (1999). Estratégias de aprendizagem e desempenho escolar: Considerações para a prática educacional. Psicologia: Reflexão e Crítica, 12(2), 361-376. DOI: $10.1590 / S 0102-79721999000200008$

Boruchovitch, E., \& Santos, A. A. A. (2015). Psychometric studies of the learning strategies scale for university students. Paidéia, 25(60), 19-27. DOI: 10.1590/198243272560201504

Carlotto, R. C., Teixeira, M. A. P., \& Dias, A. C. G. (2015). Adaptação acadêmica e coping em estudantes universitários. PsicoUSF, 20(3), 421-432. DOI: 10.1590/141382712015200305

Cheng, S., Zhang, L. F., \& Hu, X. (2016). Thinking styles and university self-efficacy among deaf, hard-of-hearing, and hearing students. Journal of Deaf Studies and Deaf Education, 21(1), 44-53. DOI: 10.1093/ deafed/env032

Cohen, J. (1988). Statistical power analysis for the behavioral sciences. Hillsdale, NJ: Erlbaum.

Cunha, N. B., \& Boruchovitch, E. (2016). Percepção e conhecimento de futuros professores sobre seus processos de aprendizagem. Pro-Posições, 27(3), 31-56. DOI: $10.1590 / 1980-6248-2015-0008$

Dembo, M. H. (1994). Applying educational psychology (5th ed.). New York: Longman.

Fan, J. (2016). The role of thinking styles in career decision-making self-efficacy among university students. Thinking Skills and Creativity, 20, 63-73. DOI: 10.1016/j. tsc.2016.03.001

Fan, J., \& Zhang, L. F. (2014). The role of perceived parenting styles in thinking styles. Learning and Individual Differences, 32, 204211. DOI: $10.1016 / j . l i n d i f .2014 .03 .004$

Fan, J., Zhang, L. F., \& Chen, C. (2018). Thinking styles: Distinct from personality? Personality and Individual Differences, 125, 50-55. DOI: $10.1016 /$ j.paid.2017.12.026

Ganda, D. R., \& Boruchovitch, E. (2015).
Self-handicapping strategies for learning of preservice teachers. Estudos em Psicologia, $32(3)$, 417-425. DOI: 10.1590/0103166X2015000300007

Igue, E. A., Bariani, I. C. D., \& Milanesi, P. V. B. (2008). Vivência acadêmica e expectativas de universitários ingressantes e concluintes. Psico-USF, 13(2), 155-164. DOI: 10.1590/ S1413-82712008000200003

Mendes, J. C. S., Fraga, A., Medeiros, C., Moniz, D., Miranda, L., \& Medeiros, T. (2018). Autoconsciência da aparência e a adaptação no ensino superior. Revista Portuguesa de Investigação Comportamental e Social, 4(2), 41-47. https://dialnet.unirioja.es/servlet/ articulo? codigo $=6572855$

Oliveira, K. L., Inácio, A. L. M., \& Buriolla, H. (2016). Diferenças considerando ano escolar no ensino fundamental: Um estudo com estilos intelectuais. Argumentos PróEducação, 1(3), 408-422. Disponível em https://www.semanticscholar.org/paper/ Diferen\%C3\%A7as-considerando-anoescolar-no-Ensino-um-Oliveira-In\%C3\%A $\underline{1 \text { cio/7b17b84ce09f0ae0985f486a309cad05c }}$ $\underline{038 \mathrm{~d} 34 \mathrm{e}}$

Oliveira, K. L., Inácio, A. L. M., Santos, A. A. A., Mariano, M. L., \& Franco, S. A. P. (2017). Estudo exploratório sobre os estilos intelectuais no ensino médio. Argumentos Pró-Educação, 2(6), 535-551. Disponível em http://ojs.univas.edu.br/index.php/ argumentosproeducacao/article/view/251

Oliveira, K. L., Santos, A. A. A., \& Inácio, A. L. M. (2018a). Adaptação acadêmica e estilos intelectuais no ensino superior. Estudos interdisciplinares em Psicologia, 9(3supl), 73-89. DOI: 10.5433/2236-6407.2018v9n3suplp73

Oliveira, K. L., Santos, A. A. A., \& Inácio, A. L. M. (2018b). Tradução, adaptação e evidências de validade do Thinking Styles Inventory - Revised II (Tsi-R2) no Brasil. Avaliação Psicológica, 17(1), 121-130. DOI: 10.15689/ap.2017.1701.13.13637 
Oliveira, K. L., Trassi, A. P., \& Santos, A. A. A. (2017). Estilos intelectuais: Revisitando e atualizando conceitos. In S. C. Martinelli \& D. C. Fernandes (Orgs.), Aprendizagem escolar na contemporaneidade (pp. 64-77). Curitiba, Brasil: Juruá.

Purdie, N., \& Hattie, J. (1996). Cultural differences in the use of strategy for selfregulated learning. American Educational Research Journal, 33, 845-871. DOI: 10.3102/00028312033004845

Santos, A. A. A., Polydoro, S. A. J., Scortegagna, S. A., \& Linden, M. S. S. (2013). Integração ao ensino superior e satisfação acadêmica em universitários. Psicologia: Ciência $e$ Profissão, 33(4), 780-793. DOI: 10.1590/ S1414-98932013000400002

Santos, A. S., Oliveira, C. T., \& Dias, A. C. G. (2015). Características das relações dos universitários e seus pares: Implicações na adaptação acadêmica. Psicologia: Teoria e Prática, 17(1), 150-163. Disponível em http://pepsic.bvsalud.org/scielo. php? script $=$ sci arttext $\&$ pid $=S 1516$ 36872015000100013\&lng=pt\&tlng=pt

Soares, A. B., \& Del Prette, Z. A. P. (2015). Habilidades sociais e adaptação à universidade: Convergências e divergências dos construtos. Análise Psicológica, 33(2), 139-151. DOI: 10.14417/ap.911

Soares, A. M., Pinheiro, M. R., \& Canavarro, J. M. P. (2015). Transição e adaptação ao ensino superior e a demanda pelo sucesso nas instituições portuguesas. Psychologica, 58(2), 97-116. DOI: 10.14195/1647-8606_58 $-2 \_6$

Sternberg, R. J. (1988). The triarchic mind: A new theory of human intelligence. New York: Viking.

Sternberg, R. J., \& Wagner, R. K. (1992). Thinking styles inventory. Unpublished manual. New Haven, CT: Yale University.

Sternberg, R. J., Wagner, R. K., \& Zhang, L. F. (2003). Thinking styles inventory - Revised I Unpublished manual. New Haven, CT: Yale
University.

Sternberg, R. J., Wagner, R. K., \& Zhang, L. F. (2007). Thinking styles inventory - Revised II. Unpublished manual. Medford/Somerville, MA: Tufts University.

Tinto, V. (1993). Leaving college: Rethinking the causes and cures of student attrition. Chicago, IL: University of Chicago Press.

Tomás, R. A., Ferreira, J. A., Araújo, A. M., \& Almeida, L. S. (2014). Adaptação pessoal e emocional em contexto universitário: $\mathrm{O}$ contributo da personalidade, suporte social e inteligência emocional. Revista Portuguesa de Pedagogia, 48(2), 87-107. Disponível em http://hdl.handle.net/1822/35646

Trassi, A. P. (2016). Estilos intelectuais, raciocínio verbal, estratégias de aprendizagem e compreensão de leitura no ensino fundamental (Dissertação de mestrado). Universidade Estadual de Londrina, Londrina, Paraná, Brasil.

Wang, T., \& Tsent, Y. (2014). Do thinking styles matter for science achievement and attitudes toward science class in male and female elementary school students in Taiwan? International Journal of Science and Mathematics Education, 13(3), 515-533. DOI: $10.1007 / \mathrm{s} 10763-013-9503-\mathrm{Z}$

Weinstein, C. E., Acee, T. W., \& Jung, J. (2011). Self-regulation and learning strategies. New Directions for Teaching \& Learning, 126, 4553. DOI: $10.1002 / \mathrm{tl} .443$

Yuan, W., Zhang, L. F, \& Fu, M. (2017). Thinking styles and academic stress coping among Chinese secondary school students. Educational Psychology, 37(8), 1015-1025. DOI: $10.1080 / 01443410.2017 .1287343$

Zhang, L. F. (2011). The developing field of intellectual styles: Four recent endeavors. Learning and Individual Differences, 21(3), 311-318. DOI: 10.1016/j.lindif.2010.11.018

Zhang, L. F. (2015). Fostering successful intellectual styles for creativity. Asia Pacific Education Review, 16(2), 183-192. DOI: $10.1007 / \mathrm{s} 12564-015-9378-5$ 
Zhang, L., \& Sternberg, R. J. (2005). A threefold model of intellectual styles. Educational Psychology Review, 17(1), 1-53. DOI: 10.1007/s10648-005-1635-4

Zimmerman, B. J., \& Martinez-Pons, M. (1986). Development of a structured interview for assessing student use of selfregulated learning strategies. American Educational Research Journal, 23(4), 614628. DOI: $10.2307 / 1163093$

\section{AgRA DECIMENTOS}

As autoras agradecem o apoio do Conselho Nacional de Desenvolvimento Científico e Tecnológico, CNPq.
INTELLECTUAL STYLES, LEARNING STRATEGIES A N D ACA DEM IC ADJUSTMENT IN BRAZILIAN HIGHER EDUCATION

\section{Abstract}

The objective of this study was to investigate how intellectual styles, learning strategies and academic adaptation are presented in higher education, seeking possible correlations and dependency relationships between these variables. Three hundred and ninety six undergraduate students from three Brazilian states $(M=22.8$ years, $S D=4.9)$, of both sexes, responded to the Intellectual/Thinking Styles Inventory - Revised II (TSI-R2), the Learning Strategies Scale (EEA-U) and the Higher Education Academic Adaptation Questionnaire (QAES). The results indicated the prevalence of hierarchical style, of social self-regulation strategies, and that students are better adapted to career planning. There was a positive and significant correlation between the hierarchical style and the adaptation to the career planning, which is of medium magnitude, and between this same style and the strategies of cognitive and metacognitive selfregulation, of small magnitude. Relationships were found between the hierarchical style and the adaptation to career planning, and between the external style and the social adaptation. The psychoeducational implications were discussed in order to conjecture deeper explanations for the difficulties of higher education students.

KEYWORDs: Learning styles; Learning strategies; Academic adjustment; Higher education 


\section{EST I L O S}

ESTRATEG I A S

A D A P T ACI Ó N

D E

I N T ELE C T UAL E S, ACADÉMICA EN LA ENSEÑANZA SUPERIOR BRASILEÑA

\section{RESUMEN}

En el presente estudio se objetivó investigar cómo se presentan los estilos intelectuales, las estrategias de aprendizaje y la adaptación académica en la enseñanza superior, siendo buscadas posibles correlaciones y relaciones de dependencia entre esas variables. Participaron 396 estudiantes de cursos de graduación de tres estados de Brasil $(M=22,8, D P=4,9)$, de ambos sexos, que respondieron al Inventario de Estilos Intelectuales/Pensamiento Revisado II (TSI-R2), a la Escala de evaluación de las Estrategias de Aprendizaje (EEA-U) y al Cuestionario de Adaptación Académica a la Enseñanza Superior (QAES). Los resultados indicaron el predominio del estilo jerárquico, de las estrategias de autorregulação social, y todavía que los estudiantes están más bien equilibrados para la proyección de carrera. Había correlación positiva y significativa entre el estilo jerárquico y la adaptación a la proyección de carrera, siendo esto de magnitud media, y entre el mismo estilo y las estrategias de autorregulação cognoscitivo y metacognitivas, de pequeña magnitud. Las relaciones de dependencia se encontraron entre el estilo jerárquico y la adaptación de planificación de carrera, y entre el estilo del estilo externo y la adaptación social. Se discutieron las implicaciones psicoeducacionais en dirección de conjeturar explicaciones más profundas de las dificultades de los estudiantes de la enseñanza superior.

Palabras Clave: Estilos de aprendizaje; Estrategias de aprendizaje; Adaptación académica; Enseñanza superior

\footnotetext{
${ }^{\mathrm{I}}$ Universidade Estadual de Londrina, Programa de Mestrado e Doutorado em Educação e Programa de Pós-Graduação em Psicologia, Paraná, Brasil. ORCID: 0000-0002-2030-500X

II Universidade São Francisco, Programa de Pós-Graduação Stricto-Sensu, São Paulo, Brasil. ORCID: 0000-0003-1892-6242

III Universidade Estadual de Londrina, Paraná, Brasil. ORCID: 0000-0001-5992-3334

IV Universidade Estadual de Londrina, Paraná, Brasil. ORCID: 0000-0001-5992-3334
}

v Universidade Estadual de Londrina, Programa de Mestrado e Doutorado em Educação, Paraná, Brasil. ORCID: 0000-00027205-744X

Toda a correspondência relativa a este artigo deve ser enviada para:

Katya Luciane de Oliveira

Laboratório de Avaliação e Pesquisa Psicológica/LAPPSIC

Depto de Psicologia e Psicanálise. Centro de Ciências Biológicas.

Universidade Estadual de Londrina. Campus Universitário

Rodovia Celso Garcia Cid (PR 445), km 380 - Cx Postal 6.001

Recebido em 30 de abril de 2018

CEP 86051-990 - Fone (43) 3371-4397

E-mail: katyauel@gmail.com 